\title{
Inverse relationship between pilus-mediated gonococcal adherence and surface expression of the pilus receptor, CD46
}

\author{
Deborah M. Tobiason and H. Steven Seifert
}

Department of Microbiology and Immunology, Northwestern University Medical School, $303 \mathrm{E}$. Chicago Ave, Searle 6-467, Chicago, IL 60611, USA
Author for correspondence: H. Steven Seifert. Tel: +1 312503 9788. Fax: +1 3125031339. e-mail: h-seifert@northwestern.edu

Pilus-mediated adherence to mucosal epithelial cells is a critical step for Neisseria gonorrhoeae to establish an infection in the human host. CD46, the defined receptor for the gonococcal pilus, is a complement-regulatory protein that is expressed on all human nucleated cells. It was observed that a piliated, Opa- variant of gonococcal strain FA1090 adhered with different efficiencies to the human epithelial cell lines tested (Chang, ME180, HEC-1B and PC-3).

Surprisingly, these differences in adherence levels did not correlate with levels of CD46 expressed by these cell lines. In fact, there was an inverse relationship between total surface-exposed CD46 and gonococcal adherence. Four major isoforms of CD46 are produced due to alternative RNA splicing of a surfaceexposed region and the cytoplasmic tail. The relative isoform surface expression of each cell line was determined, and each was found to express different ratios of the four CD46 isoforms. No correlation could be derived between CD46 isoform surface expression and pilus-mediated gonococcal adherence, indicating that CD46 does not act as a classic receptor for gonococcal pili.

Keywords: Neisseria gonorrhoeae, human epithelial cells, fluorescent labelling, flow cytometry

\section{INTRODUCTION}

The Gram-negative, obligate human pathogen Neisseria gonorrhoeae (the gonococcus) is the causative agent of the sexually transmitted disease gonorrhoea. The disease is usually limited to the urogenital tract, although ophthalmia is the most significant neonatal gonococcal infection (Handsfield et al., 1973). Therefore, several different epithelial cell lines derived from urogenital tract and conjunctival tissues have been used in studies of this organism. N. gonorrhoeae adheres to the mucosal epithelium, and a number of gonococcal surface molecules play a role in this adhesion process. Initial attachment is mediated by type IV pili, which are important for establishing infection in human volunteers (Kellogg et al., 1963; Swanson et al., 1987). After adherence via pili to epithelial cells, other surface elements such as Opa proteins and lipooligosaccharide can provide additional, stabilizing interactions with the

Abbreviations: CFDA-SE, 5- and 6-carboxyfluorescein diacetate, succinimidyl ester; FACS, fluorescence-activated cell sorter. epithelial cell surface. The bacteria then may invade and transcytose through the epithelial cell to eventually colonize the subepithelium (Merz et al., 1996). Signal transduction in the epithelial cell during infection may lead to an induction of cytokine production and hence development of a massive inflammatory response, or the infection may remain asymptomatic (reviewed by Nassif et al.,1999)

The pilin protein is the major component of the gonococcal type IV pilus. Antigenic variation of this protein has been shown to affect pilus-mediated adherence to human cells in vitro (Jonsson et al., 1994; Long et al., 1998; Rudel et al., 1992). The PilC protein is a phase-variable, minor pilus component that also plays a role in adherence to epithelial cells (Jonsson et al., 1991; Rudel et al., 1992). PilC has been localized to the tip of the pilus (Rudel et al., 1995) and to the bacterial cell surface (Rahman et al., 1997). Piliated gonococcal pilC mutants show decreased adherence to epithelial cells (Nassif \& So, 1995; Rudel et al., 1992), and purified PilC protein binds to epithelial cells in vitro (Rudel et al., 1995) and blocks adherence of N. gonorrhoeae and 
Neisseria meningitidis (Scheuerpflug et al., 1999). Both pilin and PilC have a role in pilus-mediated adherence to epithelial cells.

An epithelial cell-surface receptor for gonococcal pili is human membrane cofactor protein (MCP, CD46) as identified by Källström et al. (1997). Transfected Chinese hamster ovary cells expressing CD46 bind piliated but not non-piliated gonococci, and this adherence can be blocked with recombinant CD46 protein and $\alpha$-CD46 antibodies (Källström et al., 1997). In addition, purified neisserial pili trigger a transient increase in internal calcium levels in target epithelial cells, and the calcium flux is blocked by $\alpha$-CD46 antibodies (Källström et al., 1998), suggesting that interaction of pili with CD46 results in signal transduction. Human CD46 is a widely distributed complement regulator whose main function is to protect host cells from autologous complement attack by acting as a cofactor for proteolytic inactivation of $\mathrm{C} 3 \mathrm{~b}$ and $\mathrm{C} 4 \mathrm{~b}$ by factor I (Liszewski et al., 1991; Seya et al., 1986). CD46 has been found on every human cell and tissue type examined except for erythrocytes (Seya et al., 1990), and it is expressed at very high levels on most epithelial cell lines (Johnstone et al., 1993; McNearney et al., 1989). Due to alternative splicing, four major isoforms of CD46 are produced: $\mathrm{BC} 1, \mathrm{BC} 2, \mathrm{C} 1$ and $\mathrm{C} 2$ (Post et al., 1991). Splicing occurs within a Ser-Thr-Pro (STP)-rich region, which is surface-exposed, resulting in the presence or absence of the B portion. A second splicing event determines which of two alternative cytoplasmic tails is expressed. All four of the major CD46 isoforms can be expressed on one cell, and predominance of certain isoforms on specific tissues has been noted (Russell et al., 1992). In addition to being the gonococcal pilus receptor, CD46 is also a receptor for measles virus (Dorig et al., 1993), human herpes virus-6 (HHV-6) (Santoro et al., 1999) and M protein of group A Streptococcus pyogenes (Okada et al., 1995). Also, a significant negative correlation between CD46 expression and Helicobacter pylori infection was observed on biopsies of human gastric mucosa (Sasaki et al., 1998). Therefore, CD46 appears to be a highly conserved protein that is important to several microbial pathogens.

In this study, we developed a fluorescence-activated cell sorter (FACS)-based assay for measuring gonococcal adherence. Using this assay we observed different levels of pilus-mediated adherence to different pertinent human epithelial cell lines. Surprisingly, we found that pilus-mediated adherence has an inverse correlation to the level of surface-exposed CD46 expression on these cell lines. CD46 isoform expression was determined for each of these epithelial cell lines, but the level of isoform expression did not correlate with levels of pilusmediated adherence.

\section{METHODS}

Bacterial strains and growth conditions. Gonococci were grown on GC Medium Base (GCB; Difco) plus Kellogg supplements (Kellogg et al., 1963) at $37^{\circ} \mathrm{C}$ in $5 \% \mathrm{CO}_{2}$.
Defined pilin variants of N. gonorrhoeae FA1090 (RM11, RM11.2 and RM21) (Long et al., 1998) were used in these studies. Each pilin variant contained an IPTG-regulatable gonococcal recA allele (Seifert, 1997) to maintain a population with a stable pilE sequence. Each pilin variant was tested for Opa protein expression by Western analysis using the $\mathrm{mAb}$ 4B12 (provided by M. Blake, North American Vaccine Inc., Columbia, MD, USA), and only predominately $\mathrm{Opa}^{-}$stocks were used.

Cell lines and growth conditions. The human epithelial cell lines ME180 endocervical (ATCC HTB 33), Chang conjunctival (ATCC CCL 20.2), Hec-1B endometrial (ATCC HTB 113) and PC-3 prostate (ATCC CRL 1435), and CHOK1 Chinese hamster ovary cells (ATCC CCL 61) were maintained in RPMI 1640 (Gibco-BRL) supplemented with 5\% fetal bovine serum (Gibco-BRL), penicillin $\left(100 \mathrm{U} \mathrm{ml}^{-1}\right)+$ streptomycin $\left(100 \mu \mathrm{g} \mathrm{ml}^{-1}\right)$ (Gibco-BRL) and amphotericin B solution $\left(2.5 \mu \mathrm{g} \mathrm{ml}^{-1}\right.$; Biologs $)$ at $37^{\circ} \mathrm{C}$ in $5 \% \mathrm{CO}_{2}$. All experiments were carried out free from antibiotics and amphotericin.

Adherence assays. The standard c.f.u. adherence assay was performed essentially as described by Long et al. (1998). For the FACS-based adherence assay, $2 \times 10^{5}$ epithelial cells per well were plated in a 24 -well plate $24 \mathrm{~h}$ prior to incubation with gonococci. Eighteen hours prior to labelling, gonococci were heavily streaked onto GCB plates and incubated at $37^{\circ} \mathrm{C}$ in $5 \% \mathrm{CO}_{2}$. Gonococci were swabbed up from the plates with Dacron swabs and resuspended in liquid GCB without Kellogg supplements. Bacteria were rinsed and resuspended in incomplete PBS (Gibco-BRL) to an $\mathrm{OD}_{550}$ of 0.9 . Bacteria were labelled with 5- and 6-carboxyfluorescein diacetate, succinimidyl ester (CFDA-SE; Molecular Probes) as described by Logan et al. (1998). Excess dye was washed off, and the labelled bacteria were resuspended in RPMI 1640. Labelled bacteria were incubated with the epithelial cell monolayer at $37^{\circ} \mathrm{C}$ in $5 \% \mathrm{CO}_{2}$ for $1-2 \mathrm{~h}$ to avoid internalization of the bacteria. Monolayers were washed three times with incomplete PBS to remove non-adherent gonococci and then incubated with $1 \%$ saponin in incomplete PBS for $10 \mathrm{~min}$ at $37{ }^{\circ} \mathrm{C}$ in $5 \% \mathrm{CO}_{2}$ to disrupt the monolayer. Cells plus adherent, labelled gonococci were fixed with $1 \%$ formaldehyde in incomplete PBS. Flow cytometry (see below) was used to measure the level of fluorescence emitted by each epithelial cell, which corresponded to the relative number of adherent, fluorescing gonococci.

Analysis of CD46 levels. Epithelial cells were plated $24 \mathrm{~h}$ before harvest at a concentration of $2 \times 10^{5}$ cells per well in a $24-w e l l$ plate. At the time of harvest cells were approximately $80 \%$ confluent. However, no significant difference in CD46 expression was observed when cells were between 30 and $100 \%$ confluent. For flow cytometric analysis, cells were directly stained by incubating with FITC-conjugated $\alpha$-CD46 mAb N24 (PharMingen) or indirectly stained by incubating with $\alpha$-CD46 mAb GB24 (J. Atkinson, Washington University, St Louis, MO, USA) and FITC-conjugated polyclonal antibodies against mouse IgG (PharMingen). Flow cytometry (see below) was used to measure the level of fluorescence.

Flow cytometry. Flow cytometry was carried out on a FacsSort (Becton Dickinson) instrument with CellQuest software. Instrument settings were adjusted so that fluorescence of cells with either no bacteria or no antibody fell within the first decade of the logarithmic scale on which the emission is displayed. The mean fluorescence intensity of negative controls ranged from $2 \cdot 0$ to $4 \cdot 0$. For the adherence assays, the 
percentage of cells bound to bacteria was determined by enumerating cells fluorescing with intensity exceeding the first decade of the four-decade logarithmic scale of fluorescence intensity. The relative number of fluorescing bacteria or antibody bound per cell was expressed as the geometric mean of the histogram plotting level of fluorescence versus the number of events (epithelial cells) measured. Plots show at least 10000 events.

Fluorescence microscopy. Adherence assays were performed as described above except that epithelial cells were grown on coverslips (Sarstedt) in 24-well plates. After the final wash, coverslips were mounted onto slides and sealed with nailpolish. An Olympus IX inverted fluorescence microscope with a Photometrix cooled charge-coupled device (CCD) camera (CH350/LCCD) driven by DeltaVision software from Applied Precision was used to detect fluorescently labelled gonococci bound to epithelial cells. Approximately twenty optical sections were taken through the depth of the cell, and DeltaVision software ( $\operatorname{soft} \mathrm{WoRx}_{\mathrm{x}}$ ) was used to deconvolve these images.

Determination of CD46 isoform expression. Reverse transcription was carried out as part of the Cells-to-cDNA kit (Ambion). The 3'-UT MCP-specific antisense primer (Post et al., 1991) was used to produce CD46-specific cDNA from each human cell line. CHOK-1 cells were used as a negative control, and no human CD46 was detected. PCR was used to amplify the CD46 gene from the cDNA obtained for each cell line. Primers to constant portions of the gene were used for the amplification: 3'-UT MCP (Post et al., 1991) and 5'-MCP SCR4 (5'-GGTCAAATGTCGATTTCCAGTAGTCG-3'). Amplification products were separated on $2 \%$ Metaphor agarose (FMC Bioproducts) gels to distinguish between CD46 isoforms based upon size. As a control, the four major isoforms were amplified from plasmids containing each isoform (provided by $\mathrm{J}$. Atkinson) using the conditions described above. Gels were photographed with a digital camera (AlphaImager 2000; Alpha Inotech) and the percentage of each isoform in each cell line was determined by densitometry using ImageQuant software (Molecular Dynamics). The percentage of each isoform was normalized to the total surface-exposed CD46 for each cell line by multiplying the mean fluorescence of total CD46 by the fraction (percentage) of isoform expression measured above. Results are plotted as relative fluorescence of each isoform in each epithelial cell line.

\section{RESULTS}

\section{A FACS-based assay for measuring gonococcal adherence}

Gonococcal adherence is often measured by incubating an epithelial cell monolayer with bacteria, washing away non-adherent bacteria, removing epithelial cells bound to bacteria and plating to determine c.f.u. (Long et al., 1998). The degree of adhesion is calculated as the ratio of cell-associated c.f.u. per c.f.u. present in the inoculum, or simply as c.f.u. $\mathrm{ml}^{-1}$ recovered from the monolayer. To more accurately compare adherence of gonococcal pilin variants to different epithelial cell lines, an assay using flow cytometry was adapted from an assay originally used to examine adherence of $H$. pylori (Logan et al., 1998). Gonococci were fluorescently labelled with CFDA-SE, which resulted in uniformly

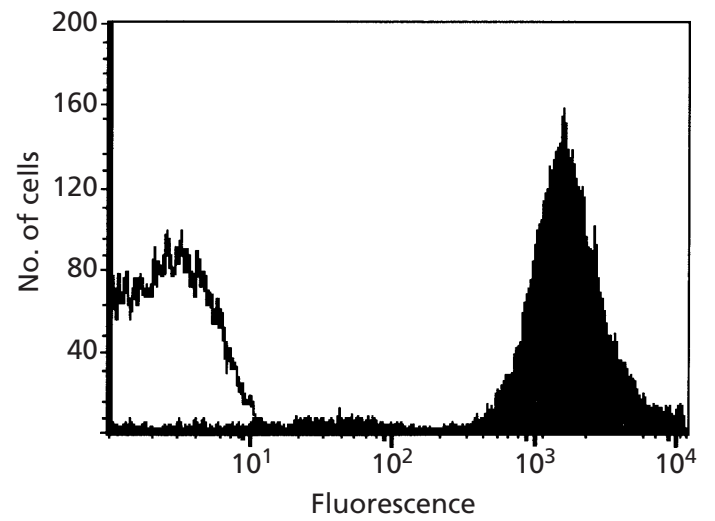

Fig. 1. N. gonorrhoeae homogeneously labelled with the fluorescent probe, CFDA-SE. The piliated FA1090 variant RM11 was labelled with CFDA-SE. Fluorescence was measured in the flow cytometer, counting 50000 cells each of labelled (black area on right) and of unlabelled (white area on left) bacteria. The resulting histogram indicates that this chemical agent labels the majority of the bacterial cells in a uniform manner, as represented by the tight peak of fluorescence.

labelled bacterial cells for each experiment (Fig. 1). CFDA-SE is a membrane-permeable ester which only fluoresces once cleaved by esterases within the cytoplasm, where it remains covalently linked to intracellular proteins via the succinimidyl group (Weston \& Parish, 1990). Cell viability was not affected by this labelling technique, and the fluorescence intensity of the labelled cells was stable for at least $24 \mathrm{~h}$ (data not shown). CFDA-SE-labelled piliated and non-piliated gonococci were incubated with human ME180 endocervical cells (Fig. 2). Non-adherent bacteria were removed by washing, and the epithelial cell monolayer plus adherent bacteria were then removed from the well for FACS analysis. A minimum of 10000 epithelial cells was measured for each sample, and the proportion of cells with adherent bacteria and the relative number of bacteria adhering to each cell was determined. The mean fluorescence intensity measured for each epithelial cell was related to the number of fluorescently labelled gonococci bound. The mean fluorescence increased in a dose-dependent manner as the number of bacteria added to the monolayer of cells increased (Fig. 2a). In addition, when the inoculum contained $5 \times 10^{6}$ labelled bacteria $($ m.o.i. $=10)$, over $95 \%$ of the epithelial cells incubated with bacteria exhibited fluorescence over background, indicating that the majority of cells were bound to gonococci; with a m.o.i. of 1 , approximately $50 \%$ of the epithelial cells showed fluorescence above background, while with a m.o.i. of $0 \cdot 1$, only $5 \%$ of the cells fluoresced above background (data not shown). Therefore, the mean fluorescence of cells with bound, labelled bacteria is a direct measurement of the number of bacteria per cell. The c.f.u. $\mathrm{ml}^{-1}$ recovered from each well was also determined for these samples (Fig. 2b). Higher numbers of piliated gonococci bound to the ME180 cells than the non-piliated isogenic variant, as has been previously 

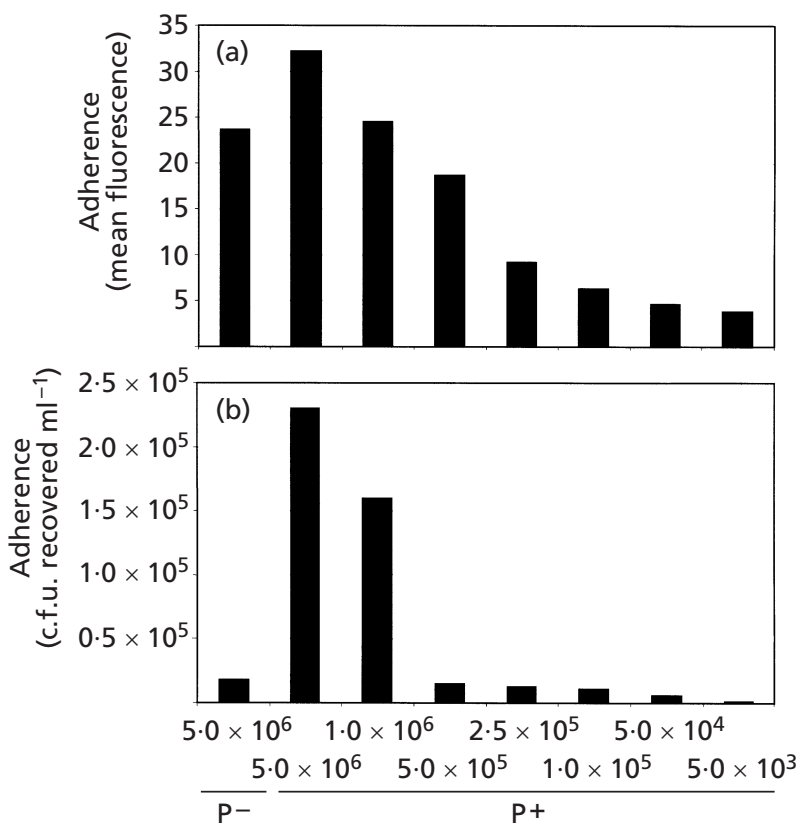

Fig. 2. Comparison of the FACS-based and the standard c.f.u. adherence assays. The piliated $\left(\mathrm{P}^{+}\right)$gonococcal FA1090 variant RM11 was labelled and incubated at various concentrations with ME180 cells. Numbers below each column indicate the number of c.f.u. incubated with the cell monolayer per well. A nonpiliated $\left(\mathrm{P}^{-}\right)$variant of $\mathrm{FA} 1090$ was used as a negative control at $5 \times 10^{6}$ bacteria per well. (a) For the fluorescence assay, the number of bacteria bound per cell is indicated by mean fluorescence. (b) For the standard c.f.u. assay, the number of adherent bacteria recovered from each well is presented as c.f.u. $\mathrm{ml}^{-1}$.

observed. With both assays, the adherence level of the non-piliated strain with an inoculum of $5 \times 10^{6}$ bacteria per well was less than that observed for the piliated gonococci with an inoculum of $1 \times 10^{6}$ bacteria per well. At lower levels of input bacteria, the FACS-based assay was more sensitive than the standard c.f.u. assay (Fig. 2). To determine whether gonococcal invasion occurred during the short incubation time of this assay, fluorescently labelled gonococci bound to the epithelial cells were examined using an inverted fluorescence microscope, and the images were clarified using deconvoluting software (data not shown). No internalized bacteria were observed. Therefore, differential invasion is not a factor in the adherence results. The FACS-based method of measuring adherence was utilized throughout the remainder of this paper.

\section{Differential adherence of piliated gonococci to pertinent epithelial cell lines}

Pilus-mediated gonococcal adherence has been studied extensively in vitro using cell lines derived from pertinent human tissues. Studies usually involve one or two cell lines, which are used to measure differences in adherence levels of gonococcal strains and mutants. Comparison of gonococcal adherence to multiple cell

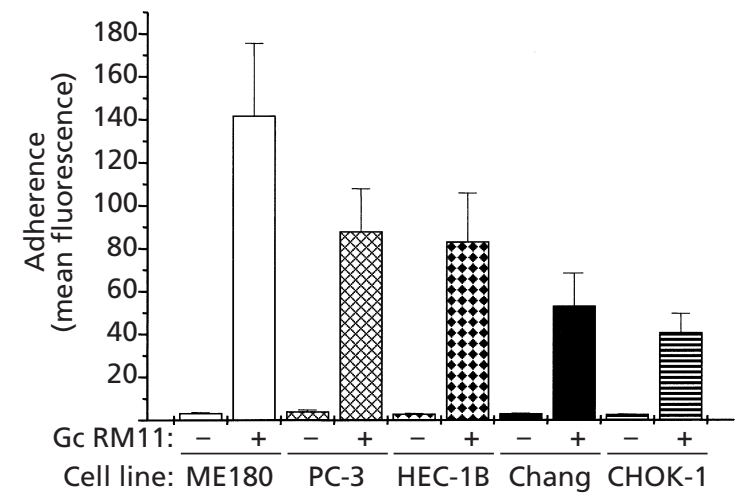

Fig. 3. Adherence of the piliated gonococcal (Gc) variant RM11 to different epithelial cell lines. Mean fluorescence indicates the mean number of bacteria per epithelial cell determined from the corresponding histogram obtained from FACS analysis. Error bars indicate the standard error from four to six independent experiments. The mean fluorescence of gonococci bound to ME180 cells versus Chang cells is significantly different based upon the Student's $t$-test $(P<0.05)$.

types has been less extensively examined (Jonsson et al., 1994; Rudel et al., 1992). To determine whether our results match previous findings and to expand upon them, we applied the FACS-based assay to examine differences in gonococcal adherence levels between pertinent cell lines. Initially, adherence of a single gonococcal strain to various epithelial cell lines was examined (Fig. 3). Four human cell lines were tested: Chang conjunctival, ME180 endocervical, Hec-1B endometrial and PC-3 prostate. Chinese hamster ovary cells (CHOK-1) were used as a non-human control cell line. A defined, piliated, $\mathrm{Opa}^{-}$FA1090 variant of $N$. gonorrhoeae (RM11) (Long et al., 1998), was used in the FACS-based adherence assay. A statistically significant difference was seen between the level of pilus-mediated adherence to Chang and ME180 cells. PC- 3 and HEC-1B cell lines had intermediate levels of bacteria bound. A non-piliated FA1090 variant had consistently lower levels of adherence to all of the cell lines examined (data not shown). Other FA1090 pilin variants (RM11.2, RM11.6 and RM21) previously described by Long et al. (1998), and a piliated variant of strain MS11 (VD300) (Koomey \& Falkow, 1987), all had intrinsically different adherence levels due to piliation states but similar relative levels of adherence to the epithelial cell lines, suggesting that differences in adherence to each cell line are not specific to one gonococcal isolate (data not shown).

\section{Levels of total surface-exposed human CD46 and variations in CD46 isoforms expressed from each cell line}

The simplest explanation for the differences in pilusmediated adherence levels seen between the various epithelial cell lines is that there are different levels of expression of the gonococcal pilus receptor (CD46) on 


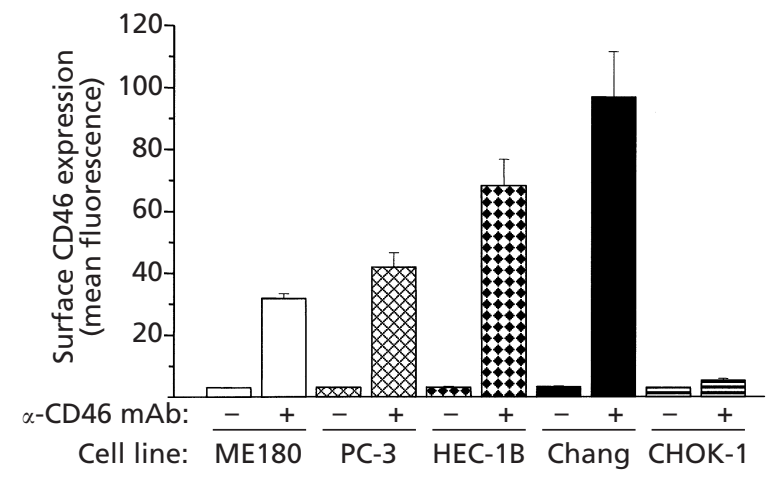

Fig. 4. Levels of surface-exposed CD46 on each cell line. The mAb N24 was used to measure CD46 expression on whole cells. Error bars indicate the standard error from four independent experiments. The mean fluorescence is significantly different between ME180 and HEC-1B, ME180 and Chang, PC-3 and HEC$1 \mathrm{~B}$, and PC-3 and Chang (Student's $t$-test, $P<0.05$ ).

each cell line. We expected that the cell lines with the most gonococci bound would have the highest level of surface-exposed CD46. To determine levels of CD46 surface expression, a FITC-conjugated $\mathrm{mAb}$ against a surface-exposed, conserved portion of human CD46 was used to detect CD46 expression on each cell line, and levels were measured by FACS analysis. Each epithelial cell line tested had significantly different overall levels of surface-exposed CD46 (Fig. 4). Surprisingly, the level of surface-exposed CD46 had an inverse correlation with the level of gonococcal adherence (Fig. 3). Indirect fluorescence staining with another anti-CD46 mAb (GB24) confirmed that the four human cell lines differ in surface-exposed levels of CD46 and verified that epitope availability for the antibody did not affect our results (data not shown). Therefore, the level of total CD46 expression on these human cells does not explain the differences in pilus-mediated gonococcal adherence.

CD46 is expressed as four major isoforms, and differential isoform expression on each cell line may explain the adherence results. Källström et al. (1998) have shown that piliated gonococci preferentially bind to the $\mathrm{BC}$ isoforms of CD46. To determine whether differential isoform expression was the basis for the observed differences in levels of gonococcal adherence, CD46 isoform-specific mRNA levels were measured. RT-PCR was performed on each cell line using a set of primers common to all CD46 isoforms, allowing for each reaction to be internally controlled. The PCR products corresponding to each isoform were separated on an agarose gel based upon size differences due to the RNA splicing events (Fig. 5a), and the percentage of each isoform in each cell line was determined using whole-band densitometry (Fig. 5b). The pattern of isoform expression was distinct for each cell line, and each cell line expressed all four isoforms. No correlation could be drawn between isoform expression and levels (a)

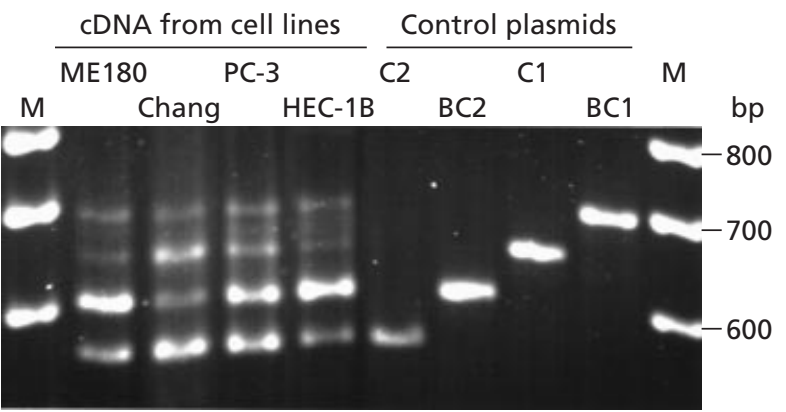

(b)

\begin{tabular}{|lcccc|}
\hline CD46 isoform & \multicolumn{4}{c|}{ Cell line: } \\
\cline { 2 - 5 } & ME180 & Chang & PC-3 & HEC-1B \\
\hline BC1 & 17 & 17 & 19 & 20 \\
C1 & 16 & 26 & 21 & 19 \\
BC2 & 40 & 20 & 27 & 37 \\
C2 & 27 & 37 & 33 & 24 \\
\hline
\end{tabular}

Fig. 5. CD46 isoform expression on each cell line. (a) Lanes on the left show the pattern of isoform expression seen for each cell line. PCR products were derived from CD46 CDNA obtained for each cell line. Lanes on the right show PCR products derived from cDNA from plasmids with known CD46 cDNA inserts representing each of the major isoforms. Lanes $M$ contain molecular mass markers. (b) Mean percentage isoform expression determined by densitometry from four to five independent experiments.

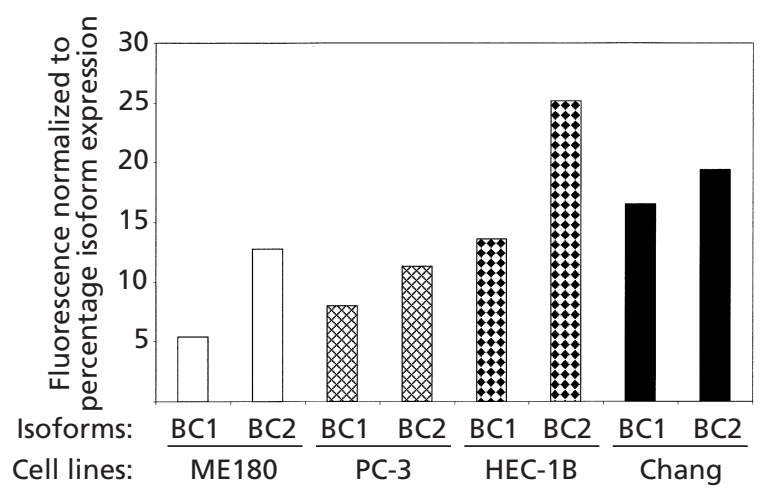

Fig. 6. Surface-exposed $B C 1$ and $B C 2$ CD46 isoforms normalized to percentage CD46 isoform expression. The mean fluorescence of total CD46 (see Fig. 4) was multiplied by the percentage of each $B C$ isoform to give the amount of surface expression for each isoform.

of gonococcal adherence to the four cell lines. In addition, the total surface-exposed CD46 measured for each cell line was normalized to the percentage of $\mathrm{BC} 1$ and $\mathrm{BC} 2$ mRNA expression (Fig. 6). Since every CD46 isoform has the same $5^{\prime}$ sequence, we assumed that all isoforms are translated equally. Also, in order to compare surface expression and mRNA expression, we assumed that each isoform is transported and expressed on the cell surface at similar levels. The calculated levels of $\mathrm{BC} 1, \mathrm{BC} 2$ or $\mathrm{BC} 1$ and $\mathrm{BC} 2$ isoform surface expression 
did not correlate with the gonococcal adherence levels on any of the cell lines.

\section{DISCUSSION}

In this study, we used a FACS-based assay to measure gonococcal adherence. The standard c.f.u. assay measures c.f.u. recovered from a well containing the epithelial cell monolayer and adherent gonococci. We modified this assay by labelling the gonococci with a probe that fluoresces once internalized and cleaved. The fluorescing probe is very stable, remains within the cytoplasm and does not affect gonococcal growth. Homogeneous labelling of gonococcal variants can be performed, allowing for accurate comparisons of fluorescence levels and number of bacteria. The flow cytometer allows for high throughput of data and a very sensitive measure of fluorescence. Differences in the number of fluorescing bacteria bound per cell are measured as the geometric mean of fluorescence in the resulting histogram. In addition, the number of cells actually bound by bacteria can be measured as the percentage of cells fluorescing above background. Doseresponse experiments indicated that the FACS-based assay is more sensitive than the standard assay when fewer bacteria are used in the inoculum (Fig. 2). This sensitivity may explain the level of fluorescence observed for the non-piliated strain versus the c.f.u. measured in the standard assay. The FACS-based assay will be a valuable, additional tool for use in gonococcal adherence studies.

Pilus-mediated adherence is a crucial step in the gonococcal infection process. Previous work by Källström et al. (1998) identified the abundant transmembrane glycoprotein CD46 as the pilus receptor. Our results demonstrate that pilus-mediated adherence varied significantly between human epithelial cell lines, in particular between ME180 endocervical cells and Chang conjunctival cells. Surprisingly, the levels of surfaceexposed CD46 had an inverse correlation to the level of pilus-mediated adherence. In fact, the Chang cells had a threefold higher level of surface-exposed CD46 compared to ME180 cells. Since the antibody used to measure CD46 levels recognized all isoforms of CD46, these experiments did not address whether differential isoform expression could account for the adherence pattern observed. Recent results reported by Källström et al. (2001) showed no difference in adherence of gonococcal strain MS11 to ME180 and Chang cells, whereas we have observed a significant difference in adherence to these cell lines using a piliated variant of strain MS11. It is important to note that different piliated variants of MS11 were used by each of our groups in gonococcal adherence assays, and piliation state is known to affect adherence (Long et al., 1998). However, pilin variants of strain FA1090 had similar relative levels of adherence to the cell lines, indicating that piliation state does not affect differences in adherence between cell lines. A previous study using the ME180, Hec1B, PC3 and Chang epithelial cell lines showed that piliated variants of MS11 can vary in adherence to ME180, Hec1B and Chang cells, with Chang cells having the least number of gonococci bound in all cases (Rudel et al., 1992). Another discrepancy between the observations of Källström et al. (2001) and our results is that the former saw no significant difference in CD46 levels between ME180 and Chang cells using a polyclonal antibody to CD46, while we observed a large difference in expression using two independent $\mathrm{mAbs}$ to CD46. Both our group and Källström et al. (2001) measured CD46 levels in a similar manner; however, specificity of the polyclonal anti-CD46 antibody versus the mAbs used in this study may contribute to the differences seen. Other factors, such as growth conditions and maintenance of the cell lines, may alter CD46 expression and explain the differences observed.

Examination of CD46 isoform levels in the epithelial cell lines used in this work revealed that each cell line had a distinct pattern of expression as measured by RT-PCR. Our RT-PCR results differ from those of Källström et al. (2001) for the ME180, Hec1B and Chang cell lines. Perhaps the cell lines alter their CD46 isoform expression. However, our results for the ME180 cell line match results previously reported by Wang et al. (2000) for percentage isoform expression, indicating that our cell line and method of mRNA detection are consistent with those of other investigators. Prior reports have shown that more gonococci adhere to the BC isoforms than to the $\mathrm{C}$ isoforms when expressed on Chinese hamster ovary cells (Källström et al., 1998). Our results indicate that the human cell line with the highest degree of adherence (ME180) predominately expresses the BC2 isoform. Also, cell lines, such as HEC-1B, with lower adherence levels express similar amounts of the BC1 and $\mathrm{BC} 2$ isoforms as ME180. In addition, we normalized the total surface-exposed CD46 to the percentage isoform expression for each cell line. The actual number of BC1 and $\mathrm{BC} 2$ isoforms possibly expressed on the surface of Chang cells is greater than on ME180 cells. If CD46 acts as a classic receptor for the pilus, then the number of piliated bacteria bound to a cell would correlate with the number of surface-exposed CD46 molecules. Our results indicate that no distinct correlation can be derived between gonococcal adherence and surface-exposed CD46 levels or CD46 isoform expression. Based upon our observations, we propose that CD46 does not act as a classic receptor in pilus-mediated adherence.

If CD46 does not act as a classic receptor for the gonococcal pilus, then what role does CD46 play in pilus-mediated adherence? Källström et al. (1998) have demonstrated signal transduction via CD46 stimulated with purified gonococcal pili. Signal transduction was measured as a calcium flux and was blocked by $\alpha$-CD 46 antibodies. CD46 has also been implicated in signalling events during measles virus infection (Karp et al., 1996). Perhaps differences in CD46-mediated signal transduction in each cell line alter gonococcal adherence levels. Cell signalling may lead to stabilization and/or promotion of gonococcal attachment to epithelial cells. Another possibility is that differential adherence of 
piliated gonococci to these cell lines is independent of CD46. Pilus-CD46 interactions may be involved in an initial, transient attachment of gonococci, but other bacterial-host interactions may be required for the stabilization of this attachment. Differences in expression of host receptors for other gonococcal molecules may explain the differences in adherence levels that we observed. For example, expression of asialoglycoproteins, which interact with gonococcal lipooligosaccharide (Porat et al., 1995), and CD66 molecules and heparan-sulfate-containing proteoglycans, which interact with Opa proteins (Chen et al., 1995; Virji et al., 1996a, b), may affect adherence to various cell lines. Our results suggest that the precise role of CD46 in gonococcal adherence still remains to be determined.

\section{ACKNOWLEDGEMENTS}

We would like to thank Darcy Blackmer and Dr John Atkinson for providing control plasmids carrying the CD46 isoforms, and Dr Milton Blake for the 4B12 antibody. We are grateful to Elizabeth Stohl for critical reading of the manuscript. This work was supported by NIH Grant RO1AI33493. D. Tobiason was partially supported by NIH Grants T32 AI07476 and F32 AI10568.

\section{REFERENCES}

Chen, T., Belland, R. J., Wilson, J. \& Swanson, J. (1995). Adherence of pilus- $\mathrm{Opa}^{+}$gonococci to epithelial cells in vitro involves heparan sulfate. J Exp Med 182, 511-517.

Dorig, R. E., Marcil, A., Chopra, A. \& Richardson, C. D. (1993). The human CD46 molecule is a receptor for measles virus (Edmonston strain). Cell 75, 295-305.

Handsfield, H. H., Hodson, W. A. \& Holmes, K. K. (1973). Neonatal gonococcal infection. I. Orogastric contamination with Neisseria gonorrhoeae. JAMA (J Am Med Assoc) 225, 697-701.

Johnstone, R. W., Loveland, B. E. \& McKenzie, I. F. (1993). Identification and quantification of complement regulator CD46 on normal human tissues. Immunol 79, 341-347.

Jonsson, A. B., Nyberg, G. \& Normark, S. (1991). Phase variation of gonococcal pili by frameshift mutation in pilC, a novel gene for pilus assembly. EMBO J 10, 477-488.

Jonsson, A. B., Ilver, D., Falk, P., Pepose, J. \& Normark, S. (1994). Sequence changes in the pilus subunit lead to tropism variation of Neisseria gonorrhoeae to human tissue. Mol Microbiol 13, 403-416.

Källström, H., Liszewski, M. K., Atkinson, J. P. \& Jonsson, A. B. (1997). Membrane cofactor protein (MCP or CD46) is a cellular pilus receptor for pathogenic Neisseria. Mol Microbiol 25, 639-647.

Källström, H., Islam, M. S., Berggren, P. O. \& Jonsson, A. B. (1998). Cell signaling by the type IV pili of pathogenic Neisseria. J Biol Chem 273, 21777-21782.

Källström, H., Blackmer Gill, D., Albiger, B., Liszewski, M. K., Atkinson, J. P. \& Jonsson, A. B. (2001). Attachment of Neisseria gonorrhoeae to the cellular pilus receptor CD46: identification of domains important for bacterial adherence. Cell Microbiol 3, 133-143.

Karp, C. L., Wysocka, M., Wahl, L. M., Ahearn, J. M., Cuomo, P. J., Sherry, B., Trinchieri, G. \& Griffin, D. E. (1996). Mechanism of suppression of cell-mediated immunity by measles virus Science 273, 228-231 (erratum Science 275, 1053).

Kellogg, D. S., Jr, Peacock, W. L., Deacon, W. E., Brown, L. \& Pirkle, C. I. (1963). Neisseria gonorrhoeae. I. Virulence genetically linked to clonal variation. J Bacteriol 85, 1274-1279.

Koomey, J. M. \& Falkow, S. (1987). Cloning of the recA gene of Neisseria gonorrhoeae and construction of gonococcal recA mutants. J Bacteriol 169, 790-795.

Liszewski, M. K., Post, T. W. \& Atkinson, J. P. (1991). Membrane cofactor protein (MCP or CD46): newest member of the regulators of complement activation gene cluster. Annu Rev Immunol 9, 431-455.

Logan, R. P., Robins, A., Turner, G. A., Cockayne, A., Borriello, S. P. \& Hawkey, C. J. (1998). A novel flow cytometric assay for quantitating adherence of Helicobacter pylori to gastric epithelial cells. J Immunol Methods 213, 19-30.

Long, C. D., Madraswala, R. N. \& Seifert, H. S. (1998). Comparisons between colony phase variation of Neisseria gonorrhoeae FA1090 and pilus, pilin, and S-pilin expression. Infect Immun 66, 1918-1927.

McNearney, T., Ballard, L., Seya, T. \& Atkinson, J. P. (1989). Membrane cofactor protein of complement is present on human fibroblast, epithelial, and endothelial cells. J Clin Invest 84, 538-545.

Merz, A. J., Rifenbery, D. B., Arvidson, C. G. \& So, M. (1996). Traversal of a polarized epithelium by pathogenic Neisseriae: facilitation by type IV pili and maintenance of epithelial barrier function. Mol Med 2, 745-754.

Nassif, X. \& So, M. (1995). Interaction of pathogenic neisseriae with nonphagocytic cells. Clin Microbiol Rev 8, 376-388.

Nassif, X., Pujol, C., Morand, P. \& Eugene, E. (1999). Interactions of pathogenic Neisseria with host cells. Is it possible to assemble the puzzle? Mol Microbiol 32, 1124-1132.

Okada, N., Liszewski, M. K., Atkinson, J. P. \& Caparon, M. (1995). Membrane cofactor protein (CD46) is a keratinocyte receptor for the $M$ protein of the group A streptococcus. Proc Natl Acad Sci US A 92, 2489-2493.

Porat, N., Apicella, M. A. \& Blake, M. S. (1995). Neisseria gonorrhoeae utilizes and enhances the biosynthesis of the asialoglycoprotein receptor expressed on the surface of the hepatic HepG2 cell line. Infect Immun 63, 1498-1506.

Post, T. W., Liszewski, M. K., Adams, E. M., Tedja, I., Miller, E. A. \& Atkinson, J.P. (1991). Membrane cofactor protein of the complement system: alternative splicing of serine/threonine/ proline-rich exons and cytoplasmic tails produces multiple isoforms that correlate with protein phenotype. J Exp Med 174, 93-102.

Rahman, M., Källström, H., Normark, S. \& Jonsson, A. B. (1997). PilC of pathogenic Neisseria is associated with the bacterial cell surface. Mol Microbiol 25, 11-25.

Rudel, T., van Putten, J. P., Gibbs, C. P., Haas, R. \& Meyer, T. F. (1992). Interaction of two variable proteins (PilE and PilC) required for pilus-mediated adherence of Neisseria gonorrhoeae to human epithelial cells. Mol Microbiol 6, 3439-3450.

Rudel, T., Scheurerpflug, I. \& Meyer, T. F. (1995). Neisseria PilC protein identified as type-4 pilus tip-located adhesin. Nature 373, 357-359.

Russell, S. M., Sparrow, R. L., McKenzie, I. F. \& Purcell, D. F. (1992). Tissue-specific and allelic expression of the complement regulator CD46 is controlled by alternative splicing. Eur J Immunol 22, 1513-1518.

Santoro, F., Kennedy, P. E., Locatelli, G., Malnati, M. S., Berger, 
E. A. \& Lusso, P. (1999). CD46 is a cellular receptor for human herpesvirus 6. Cell 99, 817-827.

Sasaki, M., Joh, T., Tada, T., Okada, N., Yokoyama, Y. \& Itoh, M. (1998). Altered expression of membrane inhibitors of complement in human gastric epithelium during Helicobacter-associated gastritis. Histopathology 33, 554-560.

Scheuerpflug, I., Rudel, T., Ryll, R., Pandit, J. \& Meyer, T. F. (1999). Roles of PilC and PilE proteins in pilus-mediated adherence of Neisseria gonorrhoeae and Neisseria meningitidis to human erythrocytes and endothelial and epithelial cells. Infect Immun 67, 834-843.

Seifert, H. S. (1997). Insertionally inactivated and inducible rec $A$ alleles for use in Neisseria. Gene 188, 215-220.

Seya, T., Turner, J. R. \& Atkinson, J. P. (1986). Purification and characterization of a membrane protein (gp45-70) that is a cofactor for cleavage of C3b and C4b. J Exp Med 163, 837-855.

Seya, T., Hara, T., Matsumoto, M. \& Akedo, H. (1990). Quantitative analysis of membrane cofactor protein (MCP) of complement. High expression of MCP on human leukemia cell lines, which is down-regulated during cell differentiation. J Immunol $145,238-245$.
Swanson, J., Robbins, K., Barrera, O., Corwin, D., Boslego, J., Ciak, J., Blake, M. \& Koomey, J. M. (1987). Gonococcal pilin variants in experimental gonorrhea. J Exp Med 165, 1344-1357.

Virji, M., Makepeace, K., Ferguson, D. J. \& Watt, S. M. (1996a). Carcinoembryonic antigens (CD66) on epithelial cells and neutrophils are receptors for Opa proteins of pathogenic neisseriae. Mol Microbiol 22, 941-950.

Virji, M., Watt, S. M., Barker, S., Makepeace, K. \& Doyonnas, R. (1996b). The N-domain of the human CD66a adhesion molecule is a target for Opa proteins of Neisseria meningitidis and Neisseria gonorrhoeae. Mol Microbiol 22, 929-939.

Wang, G., Liszewski, M. K., Chan, A. C. \& Atkinson, J. P. (2000). Membrane cofactor protein (MCP; CD46): isoform-specific tyrosine phosphorylation. J Immunol 164, 1839-1846.

Weston, S. A. \& Parish, C. R. (1990). New fluorescent dyes for lymphocyte migration studies. Analysis by flow cytometry and fluorescence microscopy. J Immunol Methods 133, 87-97.

Received 20 December 2000; revised 19 April 2001; accepted 26 April 2001. 